\title{
Calcium scoring using 64-slice MDCT, dual source CT and EBT: a comparative phantom study
}

\author{
Jaap M. Groen · Marcel J. W. Greuter · R. Vliegenthart · C. Suess · \\ B. Schmidt $\cdot$ F. Zijlstra $\cdot$ M. Oudkerk
}

Received: 11 October 2007/ Accepted: 5 November 2007/Published online: 23 November 2007

(C) The Author(s) 2007

\begin{abstract}
Purpose Assessment of calcium scoring (Ca-scoring) on a 64-slice multi-detector computed tomography (MDCT) scanner, a dual-source computed tomography (DSCT) scanner and an electron beam tomography (EBT) scanner with a moving cardiac phantom as a function of heart rate, slice thickness and calcium density. Methods and materials Three artificial arteries with inserted calcifications of different sizes and densities were scanned at rest $(0$ beats per minute) and at $50-110$ beats per minute (bpm) with an interval of $10 \mathrm{bpm}$ using 64-slice MDCT, DSCT and EBT. Images were reconstructed with a slice thickness of 0.6 and $3.0 \mathrm{~mm}$. Agatston score, volume score and equivalent mass score were determined for each artery. A cardiac motion susceptibility (CMS) index was introduced to assess the susceptibility of Ca-scoring to heart rate. In addition, a difference $(\Delta)$ index was introduced to assess the
\end{abstract}

J. M. Groen · M. J. W. Greuter (凹) · R. Vliegenthart · M. Oudkerk

Department of Radiology, University Medical Center

Groningen, University of Groningen, Groningen,

The Netherlands

e-mail: m.j.w.greuter@rad.umcg.nl

C. Suess - B. Schmidt

Siemens Medical Solutions, Forchheim, Germany

F. Zijlstra

Department of Cardiology, University Medical Center Groningen, University of Groningen, Groningen,

The Netherlands difference of absolute Ca-scoring on MDCT and DSCT with EBT. Results Ca-score is relatively constant up to $60 \mathrm{bpm}$ and starts to decrease or increase above $70 \mathrm{bpm}$, depending on scoring method, calcification density and slice thickness. EBT showed the least susceptibility to cardiac motion with the smallest average CMS-index (2.5). The average CMS-index of 64-slice MDCT (9.0) is approximately 2.5 times the average CMS-index of DSCT (3.6). The use of a smaller slice thickness decreases the CMS-index for both CT-modalities. The $\Delta$-index for DSCT at $0.6 \mathrm{~mm}$ (53.2) is approximately $30 \%$ lower than the $\Delta$-index for 64 -slice MDCT at $0.6 \mathrm{~mm}$ (72.0). The $\Delta$-indexes at $3.0 \mathrm{~mm}$ are approximately equal for both modalities (96.9 and 102.0 for 64-slice MDCT and DSCT respectively). Conclusion Ca-scoring is influenced by heart rate, slice thickness and modality used. Ca-scoring on DSCT is approximately $50 \%$ less susceptible to cardiac motion as 64-slice MDCT. DSCT offers a better approximation of absolute calcium score on EBT than 64-slice MDCT when using a smaller slice thickness. A smaller slice thickness reduces the susceptibility to cardiac motion and reduces the difference between CT-data and EBT-data. The best approximation of EBT on CT is found for DSCT with a slice thickness of $0.6 \mathrm{~mm}$.

Keywords Calcium score - Dual source CT . 64-Slice MDCT · Electron beam CT .

Heart rate 


\section{Introduction}

The presence of calcium in coronary arteries is known to be a strong indicator for coronary artery disease (CAD) [1]. It has been shown that quantification of coronary calcium enables the assessment of cardiac event risk stratification [1]. In 1990, Agatston et al. described a method which determines the amount of coronary calcium from tomographic images [2]. This method, known as the Agatston score (AS), depends on the area and the maximum CT density of the calcification detected by electron beam tomography (EBT). Since then, EBT is generally accepted as the gold standard for determining the amount of coronary calcium. Alternative scoring methods have been proposed, such as volume scoring (VS), depending on the volume of the calcification, and equivalent mass (EM) scoring, which depends on the volume and the average density of the calcification [3-5].

Calcium scoring (Ca-scoring) on EBT is known to be less susceptible to cardiac motion compared to other CT-modalities, because of its relatively high temporal resolution. However, since the appearance of multidetector computed tomography (MDCT), scanners of this type are also widely used for Ca-scoring as an alternative to EBT. Although the temporal resolution of MDCT is lower than EBT, the spatial resolution is much higher ( 0.4 vs. $1.0 \mathrm{~mm})$, enabling the detection of smaller lesions. Whereas Ca-scoring on EBT can only be used in sequential scanning mode, MDCT facilitates Ca-scoring in sequential and spiral mode. Spiral mode scanning has shown to decrease the variability of Ca-scoring when compared to sequential mode scanning [6]. With the development of dual source computed tomography (DSCT) in 2006, CT is finally approaching the temporal resolution of EBT combined with a high spatial resolution [7].

In order to use Ca-scoring as a useful diagnostic test, it must be demonstrated as accurate, clinically relevant and reproducible. Monitoring of coronary atherosclerosis by repeated scans is advocated by Callister et al. to test the response to lipid-lowering pharmacologic therapy [8] and Budoff et al. [9] showed that statin therapy induced a $61 \%$ reduction in coronary calcium progression rate. Therefore a highly reproducible scanmethod independent of in-vivo conditions to test the accuracy of Ca-scoring is desirable. In this study a cardiac phantom was used to investigate the influence of cardiac motion on the absolute Ca-score for different kinds of scanners. To our knowledge no previous study has systematically investigated the influence of the heart rate on the absolute Ca-score using EBT, 64-slice MDCT and DSCT.

The purpose of this study was therefore to assess Ca-scoring on 64-slice MDCT and DSCT versus EBT on a moving cardiac phantom as a function of heart rate, slice thickness and calcification density using 3 different Ca-scoring methods.

\section{Methods and materials}

\section{Cardiac phantom}

A moving cardiac phantom (QRM, Möhrendorf, Germany) was used to simulate the movement of the coronary arteries (Fig. 1, left) [7, 10]. The phantom consists of a robot arm which performs a pre-programmed motion (Fig. 2). The robot arm moves in a water container inside a thorax phantom (QRM, Möhrendorf, Germany) [11]. Different inserts can be attached to the robot arm. The motion curves used in this study were based on velocity curves for the LAD given in literature in order to simulate the human coronary motion as realistically as possible [12]. Three different artificial arteries were investigated which were custom built by QRM. The artificial arteries were made of hydroxyapatite (HA)
Fig. 1 Left: the cardiac phantom. Right: schematic figure of the artificial artery, the dimensions are given in millimeters
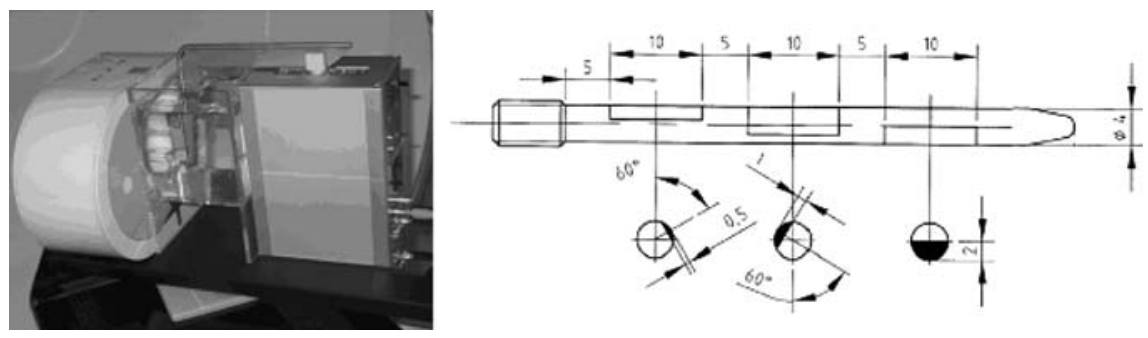


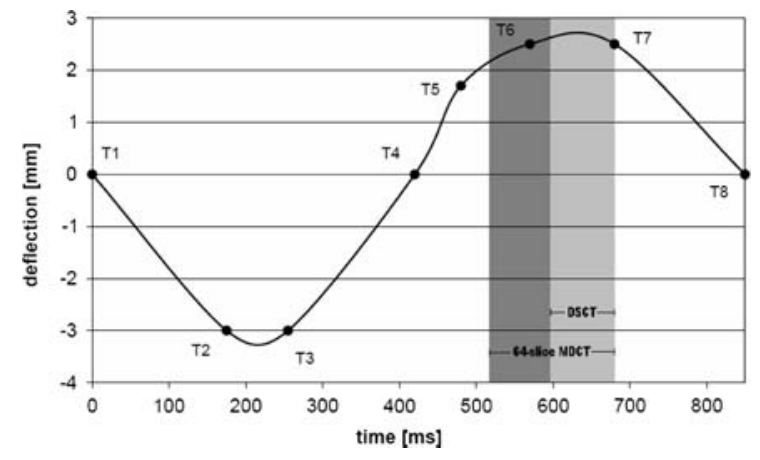

Fig. 2 Motion curve of the phantom at $70 \mathrm{bpm}$. The curve is defined by the time-deflection points T1-T8 and the reconstruction intervals of the DSCT and 64-slice MDCT are indicated by the grey areas. Other heart rates are obtained by a time scaling of the data points. For higher heart rates (>90 bpm) the data point T5 was omitted to reflect the relative larger diminishing of the diastolic phase

Table 1 The three artificial coronary arteries high, medium and low density calcification (HDC, MDC and LDC) with the properties of the inserted calcifications as specified by the manufacturer

\begin{tabular}{lll}
\hline Artificial artery & Concentration $\left(\mathrm{mgHA} / \mathrm{cm}^{3}\right)$ & Density $\left(\mathrm{g} / \mathrm{cm}^{3}\right)$ \\
\hline HDC & 796 & 1.58 \\
MDC & 401 & 1.30 \\
LDC & 197 & 1.16 \\
\hline
\end{tabular}

with a diameter of $4 \mathrm{~mm}$ and a length of $55 \mathrm{~mm}$ (Fig. 1). Each artery contained three artificial calcifications with a length of $10 \mathrm{~mm}$, a spacing of $5 \mathrm{~mm}$ and a thickness of $0.5,1.0$ and $2.0 \mathrm{~mm}$, respectively. The density of the calcifications was different in each artery, one with high density calcifications (HDC), one with medium density calcifications (MDC) and one with low density calcifications (LDC). The concentration and density of the calcifications in the three artificial atereries is given in Table 1. The artificial artery had a density of 50 Houndsfield Units (HU), simulating human blood.

\section{Data acquisition}

The phantom was positioned at an angle of 45 degrees relative to the center axis of the scanner. Every scan was repeated five times with a small random translational (approximately $2 \mathrm{~mm}$ ) and small random rotational repositioning (approximately
2 degrees) of the phantom after each scan. The ECG signal from the phantom was recorded during scanning to enable synchronization with the scanner. The scan parameters on the 64-slice MDCT (Somatom Sensation 64, Siemens, Forchheim, Germany) were: tube voltage $120 \mathrm{kV}$, tube current $250 \mathrm{mAs}$ effective, collimation $64 \times 0.6 \mathrm{~mm}$ and rotation time $330 \mathrm{~ms}$. A DSCT (Somatom Definition, Siemens, Forchheim, Germany) was used with similar scan parameters: tube voltage $120 \mathrm{kV}$, tube current $100 \mathrm{mAs} /$ rot (equivalent to the tube current of 64-slice MDCT), collimation of $64 \times 0.6 \mathrm{~mm}$ and rotation time $330 \mathrm{~ms}$. A spiral scanning mode was used on both scanners for a better reproducibility. A standard hospital calcium scoring protocol was used on the EBT-scanner ( $\mathrm{e}^{-}$Speed, GE Imatron, San Francisco, USA). This protocol uses a sequential mode with a tube voltage of $130 \mathrm{kV}$, a tube current of $44 \mathrm{mAs}$, a collimation of $3.0 \mathrm{~mm}$ and a scan speed of $50 \mathrm{~ms}$.

A standard calcium scoring kernel (B35f) was used for reconstruction of the CT-data. Images were retrospectively reconstructed with a slice thickness of $0.6 \mathrm{~mm}$ (increment $0.4 \mathrm{~mm}$ ) and $3.0 \mathrm{~mm}$ (increment $3.0 \mathrm{~mm}$ ) for both CT scanners. The phases with minimal motion were selected from the motion curves of the coronary arteries (Fig. 2) and used for reconstruction of the raw data (Table 2). The data from the EBT-scanner were reconstructed with a slice thickness of $3.0 \mathrm{~mm}$ (increment $3.0 \mathrm{~mm}$ ) at $40 \%$ of the RR-interval with a standard calcium kernel according to the standard calcium scoring protocol used in our hospital.

Ca-scoring was performed on the reconstructed image sets with commercially available software (Syngo CaScore, Siemens, Forchheim, Germany). Three different scoring methods were used: Agatston scoring, volume scoring and equivalent mass scoring. A standard scoring threshold of $130 \mathrm{HU}$ was used during the procedure. Detailed descriptions of these scoring methods can be found extensively elsewhere [4, 11, 13-15]. The three calcifications of the arteries could not be detected individually at higher heart

Table 2 Phases used for reconstruction of the images in percentage of the beat time at different heart rates used in beats per minute (bpm)

\begin{tabular}{lrrrrrrr}
\hline Heart rates (bpm) & 50 & 60 & 70 & 80 & 90 & 100 & 110 \\
64-Slice MDCT-phase (\%) & 76 & 74 & 60 & 58 & 56 & 53 & 51 \\
DSCT-phase (\%) & 83 & 82 & 70 & 69 & 69 & 67 & 66 \\
\hline
\end{tabular}


rates (at heart rates larger than $60 \mathrm{bpm}$ for 64 -slice MDCT and larger than $90 \mathrm{bpm}$ for DSCT) combined with thin slices in some of the scans. Therefore the Ca-score of the total artery was used instead of the Ca-scores of the individual calcifications.

\section{Data analysis}

Two root mean square measures were used to analyze the scoring results. The first measure quantifies the susceptibility of the calcium score to cardiac motion. The second measure quantifies the deviation of the calcium score from the reference value.

We defined a cardiac motion susceptibility (CMS) index in order to assess the susceptibility to cardiac motion of the Ca-scoring methods:

$\mathrm{CMS}=\frac{1}{N-1} \sqrt{\sum_{i=1}^{N}\left(x_{0}-x_{i}\right)^{2}} \frac{1}{x_{0}}$

in which $x_{0}$ is the Ca-scoring result at $0 \mathrm{bpm}, x_{i}$ is the scoring result at heart rate $i$ and $N$ is the total number of heart rates used. In the equation for the CMS-index a factor $1 / x_{0}$ is introduced to make the index independent of the absolute score which enables comparison of Cascores obtained at different slice thicknesses and with different scoring methods as a function of cardiac motion. A small CMS-index is equivalent to a low susceptibility of Ca-scores to cardiac motion.

A second measure was introduced to compare the calcium score results of the two CT scanners to the results of the EBT scanner. The deviation of the calcium score on $\mathrm{CT}$ versus the reference value on EBT is defined using a $\Delta$-index:

$\Delta=\sqrt{\sum_{i=1}^{N}\left(y_{i}-z_{i}\right)^{2}} \frac{1}{y_{a v}}$

in which $y_{i}$ is the EBT-score at heart rate $i, z_{i}$ is the CT-score at heart rate $i$ and $y_{a v}$ is the average EBTscore over all heart rates. The normalization factor $\mathrm{y}_{\mathrm{av}}$ was inserted to make the $\Delta$-index independent of the absolute score and to enable comparison of $\Delta$ indexes obtained with different $\mathrm{Ca}$-scoring methods and slice thicknesses. A low $\Delta$-index is equivalent to a small difference between Ca-scores on CT and EBT. The delta-index, as defined in Eq. 2, is used to quantify the difference in Agatston and volume scores on CT and EBT. For these scoring methods EBT provides the reference value. For the equivalent mass score, however, the reference value is given by the physical mass. The use of a phantom enables the possibility of calculating the true amount of calcium. Therefore the equivalent mass scoring results have been compared to the true values instead of the EBTvalues, thus $y_{i}$ is the true value and $z_{i}$ is the CT/EBTscore at heart rate $i$.

Noise levels were measured using a standard Region of Interest (ROI) technique. The ROI was placed in a section of a slice containing only water. The standard deviation of the measured HU-values within the selected ROI was considered to be a measure for the noise level.

All measurements are considered to be normally distributed. Mean and standard deviation (sd) are given for each measurement.

\section{Results}

The Ca-scoring results of the different arteries obtained with 64-slice MDCT, DSCT and EBT are shown in Fig. 3 as a function of slice thickness and heart rate using the three different scoring methods. The scoring results are relatively constant at low heart rates $(50$ $60 \mathrm{bpm}$ ). At heart rates higher than $60 \mathrm{bpm}$, however, the scores deviate from the values at lower heart rates and an increase or decrease of scoring results is observed depending on modality, slice thickness, calcification density and scoring method.

The results show a general underestimation of the Cascore for Ca-scoring obtained at $3.0 \mathrm{~mm}$ slice thickness when comparing CT-data and EBT at all heart rates except for the Agatston and volume score of the high density calcifications at 70 and $80 \mathrm{bpm}$. In general, the Ca-scores obtained with $0.6 \mathrm{~mm}$ slice thickness on CT are overestimated compared to the EBT-data or are similar to the EBT-data at all heart rates.

The scores obtained with EBT (squares) increased at heart rates above $90 \mathrm{bpm}$ for the artery containing the high density calcifications (Fig. 3, left column), whereas the artery containing the medium density calcifications remained relatively constant throughout the whole range of heart rates (Fig. 3, middle column). The artery containing the low density calcifications showed decreased scoring results at higher heart rates (Fig. 3, right column). 

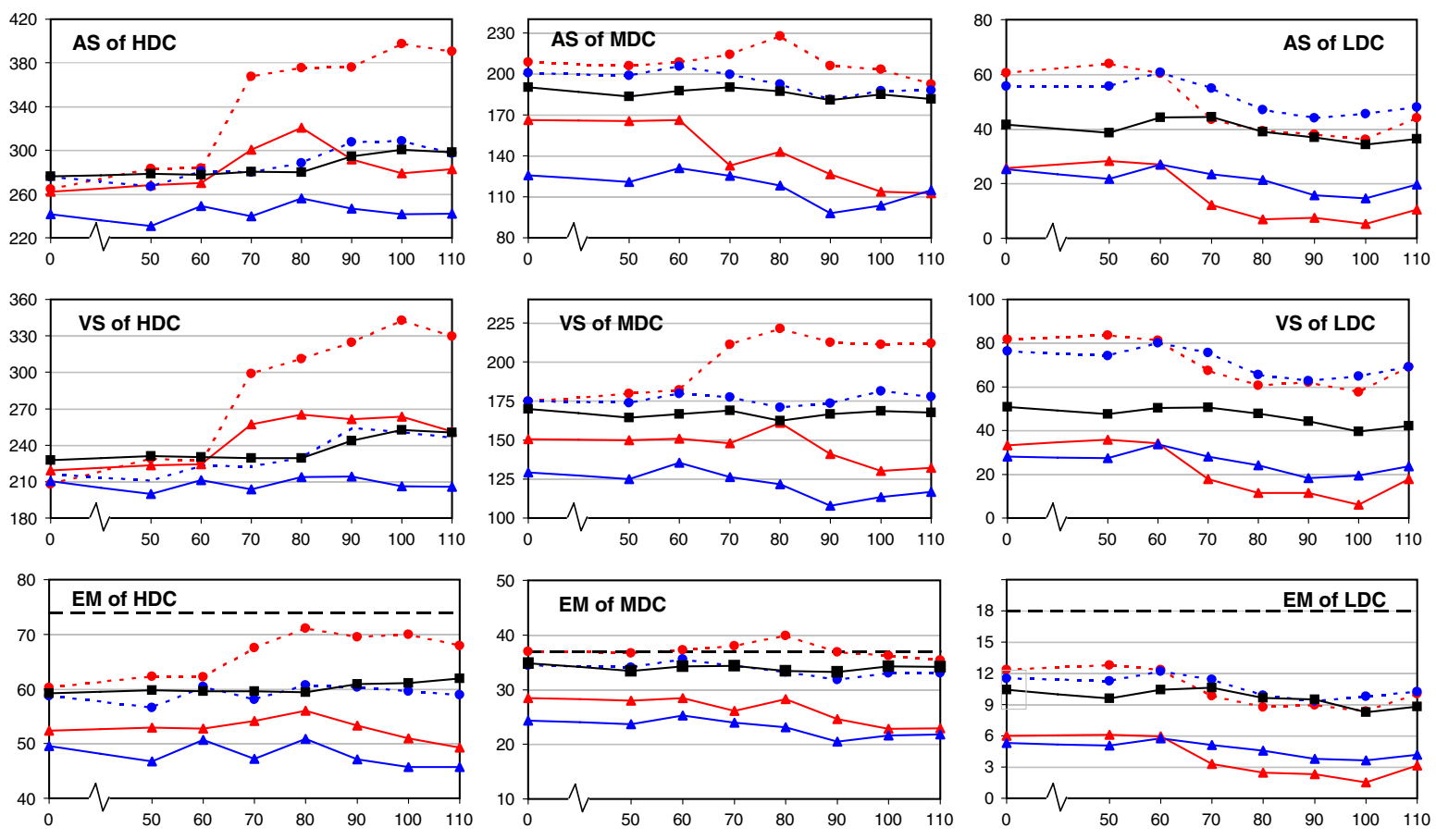

$-64 \mathrm{~S}, 0.6$

$\longrightarrow-64 S, 3.0$

$\cdots$ DS, 0.6

$\longrightarrow$ DS, 3.0

$\longrightarrow-\mathrm{EBT}, 3.0$

Fig. 3 Calcium scores as a function of heart rate in beats per minute using 64-slice MDCT at $0.6 \mathrm{~mm}$ (dotted line with circles), 64-slice MDCT at $3.0 \mathrm{~mm}$ (solid line with triangles), DSCT at $0.6 \mathrm{~mm}$ (dotted line with circles), DSCT at $3.0 \mathrm{~mm}$ (solid line with triangles), EBT (solid line with squares). Agatston score (AS), volume score (VS) and equivalent mass

The 64-slice MDCT with a slice thickness of $3.0 \mathrm{~mm}$ (solid lines with triangles) showed increased Ca-scores for the Agatston score at 70-90 bpm and for the volumes score at all heart rates for the high density calcification, whereas the equivalent mass score showed a slight decrease. The medium and low density calcification also showed a decrease in scoring results at higher heart rates.

The 64-slice MDCT with a slice thickness of $0.6 \mathrm{~mm}$ (dotted lines with circles) showed highly increased Ca-scores above $70 \mathrm{bpm}$ for the high density calcification for all scoring methods. This is also seen for the medium density calcification for the volume score, whereas the equivelnt mass and Agatston score showed a peak in Ca-scores at $80 \mathrm{bpm}$. The low density calcification showed diminished results at higher heart rates for all scoring methods.

The Ca-scores of the medium and low density calcification obtained with DSCT with a slice
(EM) score from top to bottom; artificial arteries high density calcification (HDC), medium density calcification (MDC) and low density calcification (LDC) from left to right. The thick dotted black lines in the figures in the bottom row represent the physical amount of calcium

thickness of $3.0 \mathrm{~mm}$ (solid lines with triangles) were decreased at elevated heart rates. The results of the high density calcification were relatively constant over the whole range of heart rates.

Finally DSCT at $0.6 \mathrm{~mm}$ (dotted lines with circles) showed increased results for Agatston and volume score of the high density calcification. The Agatston score of the medium density calcification showed a small decrease and relatively constant results were observed for the equivalent mass score of the high density calcification and volume and equivalent mass score of the medium density calcification. Diminishing results with increasing heart rate were observed for all methods for the low density calcification.

The influence of cardiac motion on the Ca-score (CMS-index) using the different scoring methods is calculated using Eq. 1 and is summarized in Fig. $4 \mathrm{a}-\mathrm{c}$ for all scanners and slice thicknesses. Looking at the results of the Agatston score, the average CMS-index for EBT was approximately 

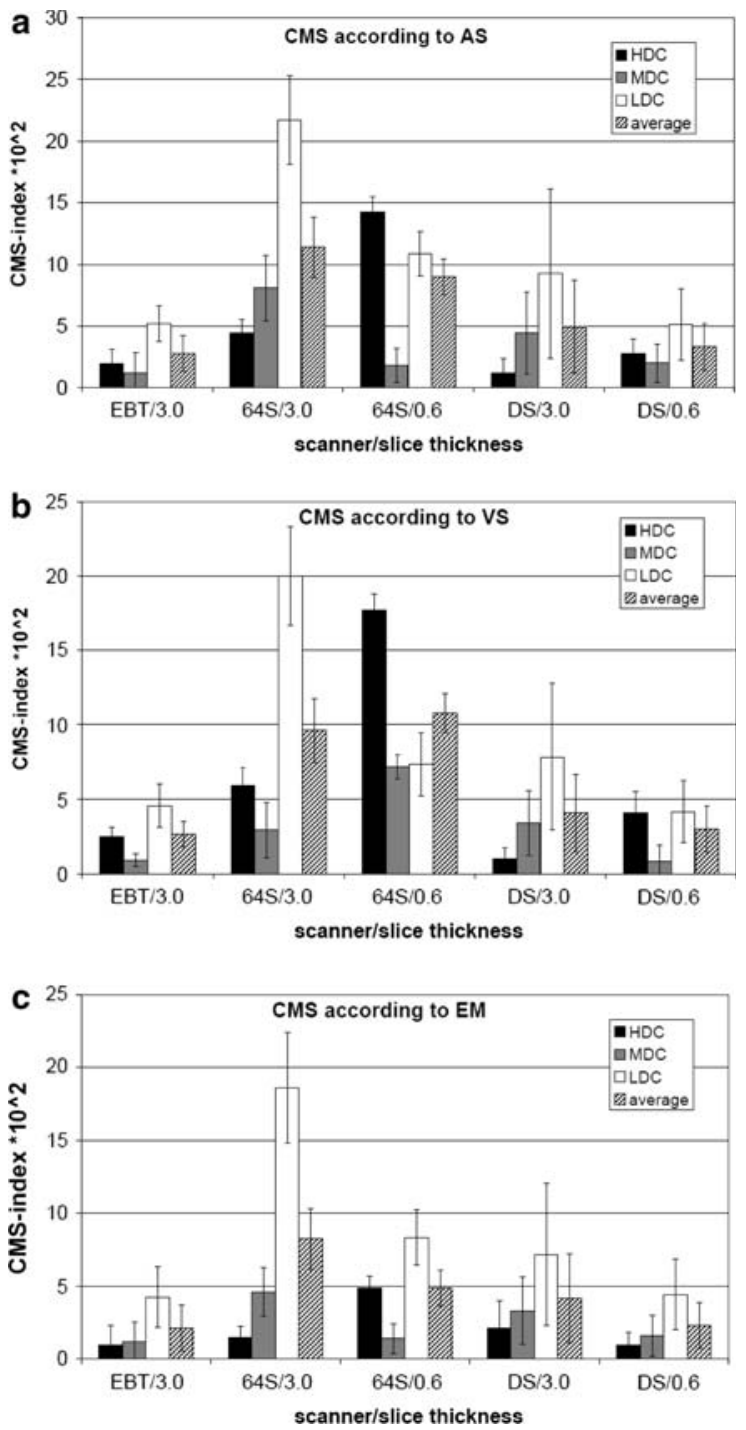

Fig. 4 Cardiac motion susceptibility-index (see text) determined with Agatston score (AS) (a), volume score (VS) (b) and equivalent mass (EM) (c) score for the high, medium, low density lesions and the average using EBT (with slice thickness of $3.0 \mathrm{~mm}$ ), 64-slice MDCT (with slice thickness of 3.0 and $0.6 \mathrm{~mm}$ ) and DSCT (with slice thickness of 3.0 and $0.6 \mathrm{~mm}$ ). A small CMS-index represents a low susceptibility to cardiac motion. The standard deviations of the CSM-index are indicated by error bars. $64 \mathrm{~S}=64$-slice MDCT; DS $=$ Dual Source CT

similar to the CMS-index of DSCT at $0.6 \mathrm{~mm}$, which was for its part approximately $60 \%$ smaller than the CMS-index of 64-slice MDCT at $0.6 \mathrm{~mm}$. The CMSindex of DSCT at $3.0 \mathrm{~mm}$ was approximately $50 \%$ higher than the CMS-index at $0.6 \mathrm{~mm}$. The CMSindex of 64-slice MDCT at $3.0 \mathrm{~mm}$ was approximately twice as large as the index of DSCT at $3.0 \mathrm{~mm}$ (Fig. 4a). The results of the susceptibility to cardiac motion using volume and equivalent mass score were similar to the results obtained with the Agatston score, except for the relatively small CMSindex for 64-slice MDCT at $0.6 \mathrm{~mm}$ using for equivalent mass score. The absolute CMS-indexes using equivalent mass were approximately $10 \%$ lower compared to the other two methods. The CMS-indexes averaged over scoring method, slice thickness and calcification density were 2.5 for EBT, 3.6 for DSCT and 9.0 for 64-slice MDCT.

The difference between the scoring results using Agatston and volume score of 64-slice MDCT and DSCT compared to EBT are calculated using Eq. 2 and are shown in Fig. 5a-b. For Agatston score (Fig. 5a), the best $\Delta$-index was observed for DSCT with a slice thickness of $0.6 \mathrm{~mm}$ (35.9 \pm 10.0 averaged over all densities). A $\Delta$-index approximately twice as large was observed for 64 -slice MDCT at $0.6 \mathrm{~mm}(65.7 \pm 9.0$ averaged over all densities). Both CT-modalities at $3.0 \mathrm{~mm}$ had a $\Delta$-index approximately two times the $\Delta$ index of DSCT at $0.6 \mathrm{~mm} \quad(91.0 \pm 10.1$ and $88.4 \pm 9.1$ for DSCT and 64-slice MDCT respectively averaged over all densities). Comparable results were observed for the volume score measurement (Fig. 5b), although the $\Delta$-indexes for the measurements at $0.6 \mathrm{~mm}$ were higher with the highest $\Delta$-index for 64 slice MDCT at $0.6 \mathrm{~mm}$.

A $\Delta$-index was calculated for all scanners comparing the equivalent mass results to the theoretical true values. The results are shown in Fig. 5c. The smallest $\Delta$-index was observed for 64 -slice MDCT $(55.9 \pm 6.8)$ followed by higher indexes for DSCT $(68.3 \pm 8.3)$ and EBT $(71.3 \pm 7.9)$ both with a slice thickness of $0.6 \mathrm{~mm}$, however the indexes of 64-slice MDCT and DSCT and the indexes of EBT and DSCT are within each margins of error shown by the error bars. Both CT-modalities at $3.0 \mathrm{~mm}$ showed $\Delta$ indexes approximately twice as large compared to the results at $0.6 \mathrm{~mm}(140.1 \pm 7.8$ and $131.1 \pm 8.5$ for DSCT and 64-slice MDCT respectively averaged over all densities).

The $\Delta$-indexes were 53.2 for DSCT and 72.0 for 64-slice MDCT both with a slice thickness of $0.6 \mathrm{~mm}$ averaged over the scoring methods and densities. The $\Delta$-indexes at $3.0 \mathrm{~mm}$ were 102.0 for DSCT and 96.9 for 64-slice MDCT averaged over the scoring methods and densities. 

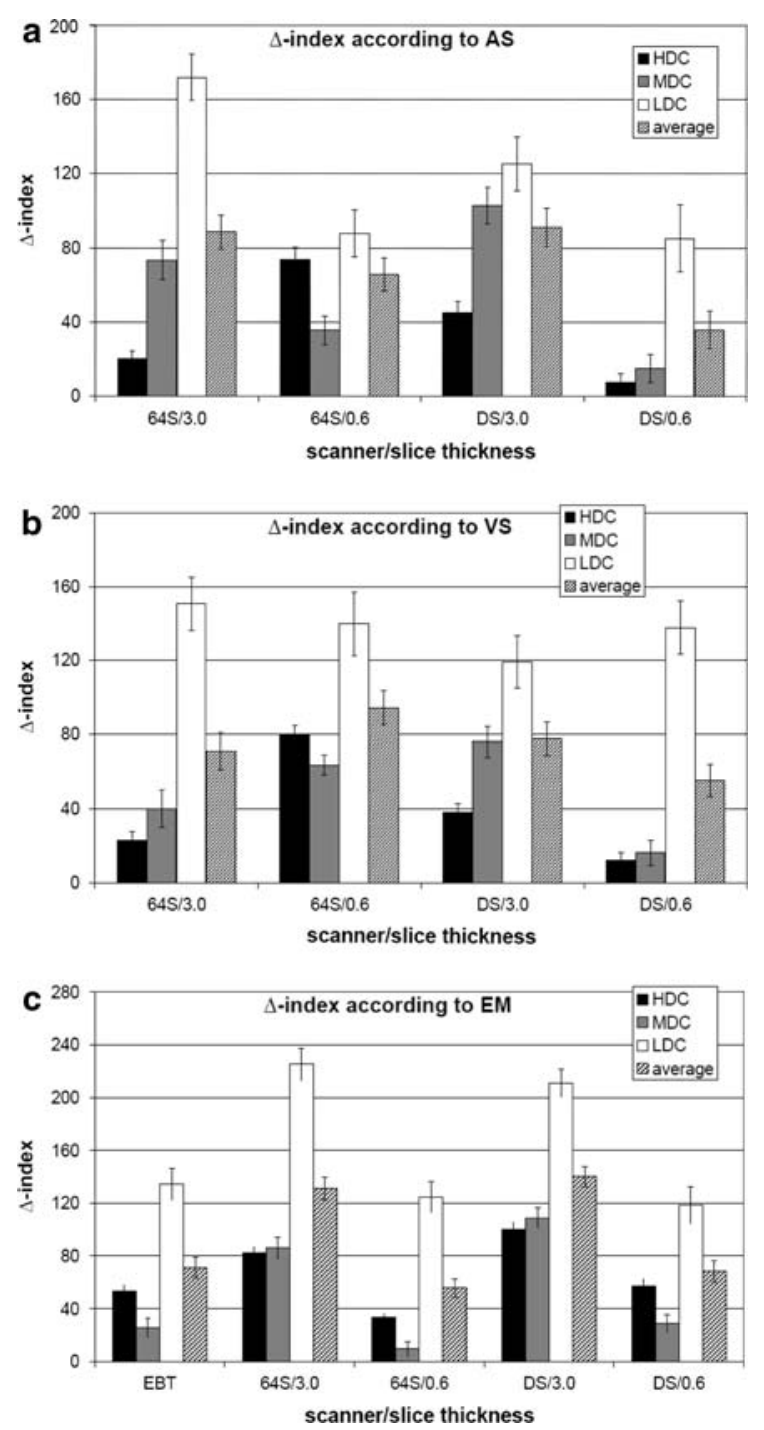

Fig. $5 \Delta$-index (see text) determined with Agatston score (AS) (a), volume score (VS) (b) and equivalent mass (EM) (c) score for the high, medium, low density lesions and the average using 64-slice MDCT and DSCT, both with slice thicknesses of 3.0 and $0.6 \mathrm{~mm}$. For Agatston and volume score, EBT has been used as a reference value ( $a$ and $b$, respectively), whereas for equivalent mass score the physical mass has been used as a reference value (c). The equivalent mass measurement includes the EBT as well. A small $\Delta$-index represents a good correspondence with the EBT results. $64 \mathrm{~S}=64$-slice MDCT; DS = Dual Source CT

Noise levels were as follows: 64-slice MDCT showed $36.1 \pm 2.9 \mathrm{HU}$ and $13.2 \pm 1.2 \mathrm{HU}$ for 0.6 and $3.0 \mathrm{~mm}$ slice thickness, respectively. DSCT showed $43.0 \pm 1.6 \mathrm{HU}$ and $16.1 \pm 1.0 \mathrm{HU}$ for 0.6 and $3.0 \mathrm{~mm}$ slice thickness, respectively. EBT with a slice thickness of $3.0 \mathrm{~mm}$ showed a noise level of $20.5 \pm 0.8 \mathrm{HU}$. The noise did not vary at different heart rates.

\section{Discussion}

An assessment was made of Ca-scoring on 64-slice multi-detector computed tomography and dual-source computed tomography versus electron beam tomography on a moving cardiac phantom as a function of heart rate, slice thickness and calcification density using 3 different Ca-scoring methods. From the results it can be concluded that the Agatston, volume and equivalent mass scores depend on heart rate, slice thickness and the CT-system used. Furthermore DSCT is approximately $50 \%$ less susceptible to cardiac motion as 64-slice MDCT in Ca-scoring.

It has been shown in previous studies that the amount of calcium in coronary arteries is generally underestimated in MDCT with respect to the gold standard EBT. Stanford et al. showed an underestimation of coronary calcium with 4-slice MDCT compared to EBT [16] and the same effect was reported by Horiguchi et al. using 16-slice MDCT $[14,17]$. Our results showed underestimation as well, but only for $3.0 \mathrm{~mm}$ slice thickness, whereas $0.6 \mathrm{~mm}$ showed an overestimation at all heart rates.

Surprisingly the Agatston scores of the medium density calcification at rest using $3.0 \mathrm{~mm}$ slices are different for the 64-MDCT and DSCT, while similar scores are expected (approximately 165 for 64-slice MDCT and 135 for DSCT). The same effect is observed for heart rates of 50 and $60 \mathrm{bpm}$. A possible explanation for this phenomenon lies within the scoring algorithm of the Agatston score. For each calcification the maximum $\mathrm{HU}$ value within the calcification is obtained. Based on this maximum value the area of the calcification is multiplied by a weighting factor. For a maximum of more than 400 $\mathrm{HU}$ this factor is 4, for a maximum between $300 \mathrm{HU}$ and $400 \mathrm{HU}$ this factor is 3 [2]. The medium density calcification has a CT density of $400 \mathrm{HU}$. The difference in scoring results can be explained by a small difference in HU between the two scanners. Where the maximum CT density within the medium density calcification could be over $400 \mathrm{HU}$ using the 64-slice MDCT, the maximum CT might have been below 400 using the DSCT. If this explanation is 
applied, the score obtained using 64-slice MDCT is more similar to the score obtained using DSCT $\left(165^{*} 3 / 4=124\right)$. Although the difference in $\mathrm{HU}$ is very small, the weighting factor of the Agatston algorithm can cause a large difference in scoring result.

At heart rates above $70 \mathrm{bpm}$ Agatston, volume and equivalent mass score differ from the results at rest and at low heart rates. This difference depends on the density of the calcification as can be seen from Fig. 3: calcifications with a high density show elevated scoring results, whereas low density calcifications are associated with diminished scoring results. A decrease of Agatston and equivalent mass score on increased heart rates using a calcification of $400 \mathrm{HU}$ has also been reported by Ulzheimer et al. using a 4slice MDCT in accordance with our results [11]. We considered the influence of image blurring on the $\mathrm{Ca}$ score as a function of the calcification density in Fig. 6 for a possible explanation for this effect. Two calcifications of identical size are shown by black lines, one with a high density (X) and one with a low density (Y). The corresponding apparent images at a relatively low and high heart rate are given by the solid grey and dotted grey line, respectively. In addition, the default Ca-scoring threshold of $130 \mathrm{HU}$ is shown by the dotted black line. At the level of the threshold the apparent width at high heart rates is larger than the apparent width at low heart rates for the high density object. The reverse effect is observed for the low density object; at high heart rates the apparent size is reduced compared to the apparent

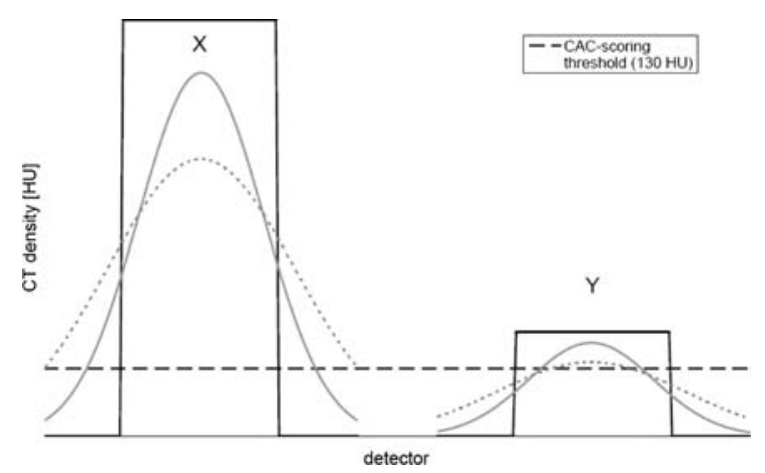

Fig. 6 Theoretical estimated CT profiles for two objects (black) with high (X) and low density (Y) exhibiting a relatively low (solid grey) and high (dotted grey) movement. The dotted black line represents the standard Ca-scoring threshold of $130 \mathrm{HU}$ size at low heart rates. From this analysis it can be concluded that at high heart rates the apparent volume of high density objects is increased and the apparent volume of low density objects is decreased. With this model we can explain the increase of calcium score on increasing heart rate for high density calcifications, and a decrease of calcium score on increasing heart rate for low density calcifications, as observed in Fig. 3. Decreasing scoring results with increasing movement have previously been reported on 4-slice CT [15].

The susceptibility of calcium score on heart rate has been assessed by the CMS-index using the 3 different scoring methods available. The results show that the CMS-index of EBT is the lowest for all methods. Therefore it can be concluded that EBT is the least susceptible to cardiac motion. The CMSindex of DSCT is approximately half the CMS-index of 64-slice MDCT, showing a reduction of $50 \%$ of the influence of cardiac motion on Ca-scoring on DSCT with respect to 64-slice MDCT. These results can be explained with the improved temporal resolution of DSCT compared to 64-slice MDCT (83 vs. $165 \mathrm{~ms}$ ). A reduction of the slice thickness also results in a lower CMS-index. Therefore we conclude that the use of a small slice thickness reduces the susceptibility to cardiac motion for both 64-slice MDCT and DSCT.

The difference between CT-data and EBT-data has been assessed by the $\Delta$-index using the Agatston and volume score, the equivalent mass results have been compared to the physical amount of calcium. The results show the lowest $\Delta$-index for DSCT with a slice thickness of $0.6 \mathrm{~mm}$ for Agatston and volume score. The CT modalities at $0.6 \mathrm{~mm}$ and EBT showed similar $\Delta$-indexes for the approximation to the physical mass. A reduction of the $\Delta$-index was observed comparing the two CT-modalities at $0.6 \mathrm{~mm}$ and $3.0 \mathrm{~mm}$. The best resemblance between EBT and CT was observed for DSCT with a slice thickness of $0.6 \mathrm{~mm}$.

The use of a smaller slice thickness has some disadvantages although it was beneficial to the scoring results in this phantom study. The noise measurements showed increased noise levels for the $0.6 \mathrm{~mm}$ slices compared to $3.0 \mathrm{~mm}$ slices. It is expected that for patient scanning the noise levels at $0.6 \mathrm{~mm}$ are too high to guarantee a reliable outcome of the Ca-scoring. To overcome these increased noise 
levels the tube current can be increased. However, this increases the patient dose as well. Although dosereduction techniques have been investigated leading to dose-reductions up to 57\% [18-21], a good balance between patient dose and accuracy of calcium scoring needs to be found.

\section{Limitations}

The EBT-data acquisition of this study was performed with a standard hospital protocol using a tube voltage of $130 \mathrm{kV}$, whereas CT scanning was performed with a tube voltage of $120 \mathrm{kV}$. Although higher energies tend to show less density, Nelson et al. reported very small differences between EBT at $130 \mathrm{kV}$ and CT at $120 \mathrm{kV}$ [22]. Therefore we expect that the influence of the difference in tube voltage can be neglected.

The pre-programmed movement of the calcified coronary arteries was 1-dimensional in contrast with the in vivo situation where the motion of human coronary arteries is 3-dimensional and the direction and orientation of the human coronary arteries can vary. In our study the movement of the calcified coronary arteries was in the $(\mathrm{x}, \mathrm{z})$ plane with a $45^{\circ}$ angle relative to the $z$-direction of the scanner. We expect that movement more perpendicular to the $\mathrm{z}$ direction of the scanner will cause more blurring in the $(\mathrm{x}, \mathrm{y})$ plane and reduce blurring in the $\mathrm{z}$-direction. In addition, we expect that movement more parallel to the z-direction of the scanner will be more subject to partial volume effects when using thick slices. Thin slices will be less subject to the PVE due to the isotropic resolution of $0.6 \mathrm{~mm}$. The motion of the robot arm was programmed according to patient data [11] and therefore we expect that our analysis shows a good correspondence with a clinical situation, but a clinical validation is advocated.

The coronary artery we used for our simulation, the LAD, exhibits lesser motion than the LCX and especially the RCA, which exhibits very large motion swings especially in systole. In our study we, however, wanted to show the influence of motion on the coronary calcium score independent of a specific major coronary artery. We therefore have used motion curves with velocities similar to the $\mathrm{LAD}$ to simulate the motion, because if a dependency of calcium score on coronary motion could be proven for the lowest velocity of the
$\mathrm{LAD}$, we expect an even stronger dependence for the higher velocities of the LCX and RCA. In our study we have shown that for higher heart rates the under- or overestimation of the calcium score increases as a function of calcification density, independently of the absolute velocity of the artery, but depending on the relative heart rate difference from $0 \mathrm{bpm}$. Because this motion dependent effect is pronounced visible for the relative low velocity of the $\mathrm{LAD}$, we expect that the results can also be applied to the vaster moving other major arteries.

\section{Conclusion}

The results of Ca-scoring are influenced by heart rate, slice thickness and modality used. DSCT is approximately $50 \%$ less susceptible to cardiac motion than 64slice MDCT using a robot phantom. Susceptibility is further reduced with a smaller slice thickness. DSCT gives a better approximation of the absolute calcium score on EBT than results obtained with 64-slice MDCT when using a smaller slice thickness $(0.6 \mathrm{~mm})$. The two modalities show similar results when using larger slice thicknesses $(3.0 \mathrm{~mm})$. In general, the use of a smaller slice thickness further reduces the difference between CT-data and EBT-data. The best approximation to the physical amount of calcium was found using a small slice thickness, where 64-slice MDCT and DSCT show similar results. The best approximation of Ca-scoring on EBT is observed for DSCT with a slice thickness of $0.6 \mathrm{~mm}$.

Open Access This article is distributed under the terms of the Creative Commons Attribution Noncommercial License which permits any noncommercial use, distribution, and reproduction in any medium, provided the original author(s) and source are credited.

\section{References}

1. Schmermund A, Mohlenkamp S, Erbel R (2003) Coronary artery calcium and its relationship to coronary artery disease. Cardiol Clin 21(4):521-534

2. Agatston AS, Janowitz WR, Hildner FJ et al (1990) Quantification of coronary artery calcium using ultrafast computed tomography. J Am Coll Cardiol 15(4):827-832

3. Callister TQ, Cooil B, Raya SP et al (1998) Coronary artery disease: improved reproducibility of calcium scoring with an electron-beam CT volumetric method. Radiology 208(3):807-814 
4. Hoffmann U, Kwait DC, Handwerker J et al (2003) Vascular calcification in ex vivo carotid specimens: precision and accuracy of measurements with multi-detector row CT. Radiology 229(2):375-381

5. Hoffmann U, Siebert U, Bull-Stewart A et al (2006) Evidence for lower variability of coronary artery calcium mineral mass measurements by multi-detector computed tomography in a community-based cohort - consequences for progression studies. Eur J Radiol 57(3):396-402

6. Horiguchi J, Shen Y, Akiyama Y et al (2006) Electron beam CT versus 16-slice spiral CT: how accurately can we measure coronary artery calcium volume? Eur Radiol 16(2):374-380

7. Flohr T, McCollough C, Bruder H et al (2006) First performance evaluation of a dual-source CT (DSCT) system. Eur Radiol 16:256-268

8. Callister TQ, Raggi P, Cooil B et al (1998) Effect of HMG-CoA reductase inhibitors on coronary artery disease as assessed by electron-beam computed tomography. N Engl J Med 339:1972-1978

9. Budoff MJ, Lane KL, Bakhsheshi H et al (2000) Rates of progression of coronary calcium by electron beam tomography. Am J Cardiol 86:8-11

10. Schlosser T, Scheuermann T, Ulzheimer S et al (2007) In vitro evaluation of coronary stents and in-stent stenosis using a dynamic cardiac phantom and a 64-detector row CT scanner. Clin Res Cardiol 96:(online first)

11. Ulzheimer S, Kalender W (2003) Assessment of calcium scoring performance in cardiac computed tomography. Eur Radiol 13:484-497

12. Achenbach S, Ropers D, Holle J et al (2000) In-plane coronary arterial motion velocity: measurement with electron-beam CT. Radiology 216:457-463

13. Ohnesorge B, Flohr T, Fischbach R et al (2002) Reproducility of coronary calcium quantification in repeat examinations with retrospectively ECG-gated multisection spiral CT. Eur Radiol 12:1532-1540
14. Horiguchi J, Yamamoto H, Akiyama Y et al (2005) Variability of repeated coronary artery calcium measurements by 16-MDCT with retrospective reconstruction. AJR 184:1846-1917

15. Brown S, Hayball M, Coulden R (2000) Impact of motion artefact on the measurement of coronary calcium score. $\mathrm{Br}$ J Radiol 73:956-962

16. Stanford W, Thompson B, Burns T et al (2004) Coronary artery calcium quantification at multi-detector row helical CT versus electron-beam CT. Radiology 230:397-402

17. Horiguchi J, Shen Y, Akiyama Y et al (2005) Electron beam CT versus 16-MDCT on the variability of repeated coronary artery calcium measurements in a variable heart rate phantom. AJR 185:995-1000

18. Mühlenbruch G, Hohl C, Das M, Wildberger JE, Suess C, Klotz E, Flohr T, Koos R, Thomas C, Günther RW, Mahnken AH (2007) Evaluation of automated attenuationbased tube current adaptation for coronary calcium scoring in MDCT in a cohort of 262 patients. Eur Radiol 17(7):1850-1857

19. Horiguchi J, Yamamoto H, Hirai N et al (2006) Variability of repeated coronary artery calcium measurements on lowdose ECG-gated 16-MDCT. AJR 187:W1-W6

20. Moselewski F, Ferencik M, Achenbach S et al (2006) Threshold-dependent variability of coronary artery calcification measurements - implications for contrastenhanced multi-detector row-computed tomography. Eur $\mathbf{J}$ Radiol 57:390-395

21. Thomas CK, Muhlenbruch G, Wildberger JE et al (2006) Coronary artery calcium scoring with multislice computed tomography: in vitro assessment of a low tube voltage protocol. Invest Radiol 41(9):668-673

22. Nelson JC, Kronmal RA, Carr JJ et al (2005) Measuring coronary calcium on CT images adjusted for density differences. Radiology 235(2):403-414 